In Situ

Revue des patrimoines
In Situ

Revue des patrimoines

$13 \mid 2010$

Art rupestre : la 3D un outil de médiation du réel invisible?

\title{
De la manipulation des images 3D
}

Geneviève Pinçon, Camille Bourdier, Oscar Fuentes et Aurélie Abgrall

\section{(2) OpenEdition \\ Journals}

Édition électronique

URL : http://journals.openedition.org/insitu/6814

DOI : 10.4000/insitu.6814

ISSN : 1630-7305

Éditeur

Ministère de la culture

Référence électronique

Geneviève Pinçon, Camille Bourdier, Oscar Fuentes et Aurélie Abgrall, «De la manipulation des images 3D », In Situ [En ligne], 13 | 2010, mis en ligne le 16 avril 2012, consulté le 20 avril 2019. URL : http:// journals.openedition.org/insitu/6814 ; DOI : 10.4000/insitu.6814

Ce document a été généré automatiquement le 20 avril 2019.

\section{(c) (i) () $\Theta$}

In Situ Revues des patrimoines est mis à disposition selon les termes de la licence Creative Commons Attribution - Pas d'Utilisation Commerciale - Pas de Modification 4.0 International. 


\title{
De la manipulation des images 3D
}

\author{
Geneviève Pinçon, Camille Bourdier, Oscar Fuentes et Aurélie Abgrall
}

Ces dernières années, les technologies 3D ont pris une place majeure dans les études de sites d'art pariétal paléolithique, avec un recours croissant au capteur laser tridimensionnel dans l'acquisition des données (topographie, œuvres). Dans le cadre des recherches que nous conduisons sur les abris sous roche ornés magdaléniens du Roc-auxSorciers (Angles-sur-l'Anglin, Vienne) et de la Chaire-à-Calvin (Mouthiers-sur-Boëme, Charente), le rôle des technologies 3D a été étendu à la phase d'analyse des œuvres, qu'elles contribuent à enrichir formidablement.

\section{Un enregistrement fidèle et précis des œuvres pariétales paléolithiques}

Figure 1

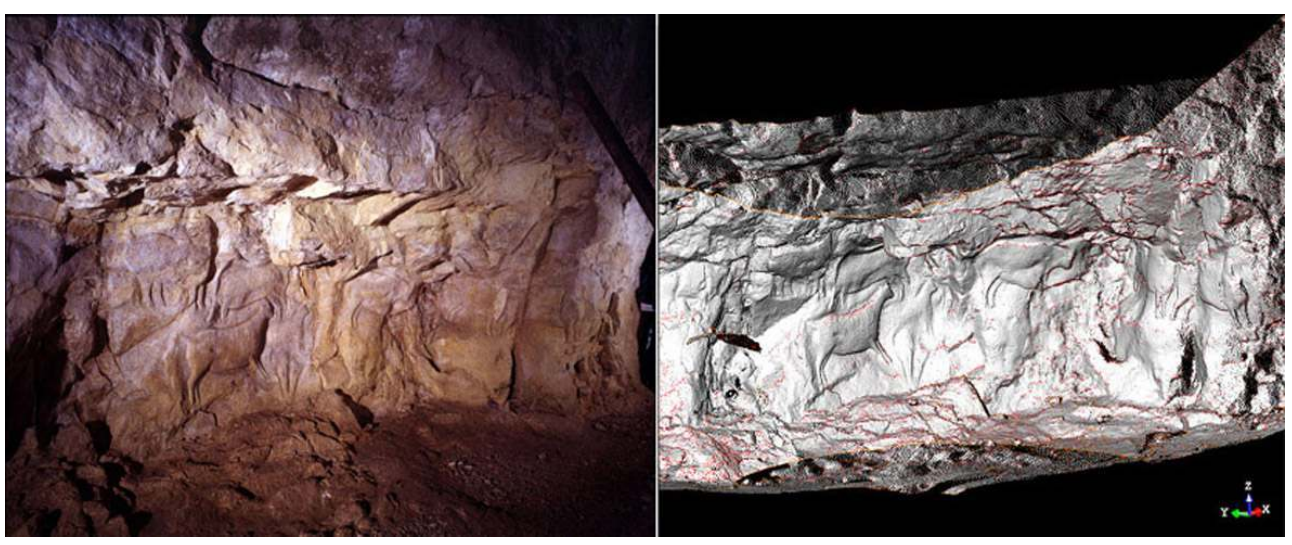

Détail de la frise sculptée du Roc-aux-Sorciers, le panneau des bouquetins : cliché et modèle numérique 3D

(C) Ministère de la Culture et de la Communication, base de données 3D Arts graphiques et patrimoine. Phot. Alain Maulny 
2 Sur les sites du Roc-aux-Sorciers et de la Chaire-à-Calvin, le recours au laser 3D a été motivé par l'enregistrement des figures, sculptées et gravées ${ }^{12}{ }^{2}$. Il fut initié dès 1999 au Roc-aux-Sorciers (fig. $\mathbf{n}^{\circ} \mathbf{1}$ ) dont l'enregistrement de la topographie et des œuvres s'est poursuivi jusqu'en 2006, avec le soutien financier de l'État, de la Région Poitou-Charentes et du Conseil général de la Vienne. La Chaire-à-Calvin (fig. $\mathbf{n}^{\circ} \mathbf{2}$ ) a bénéficié du même type enregistrement en 2006, lors de la reprise de l'étude de l'art pariétal par G. Pinçon, grâce à l'investissement du Conseil général de la Charente.

Figure 2

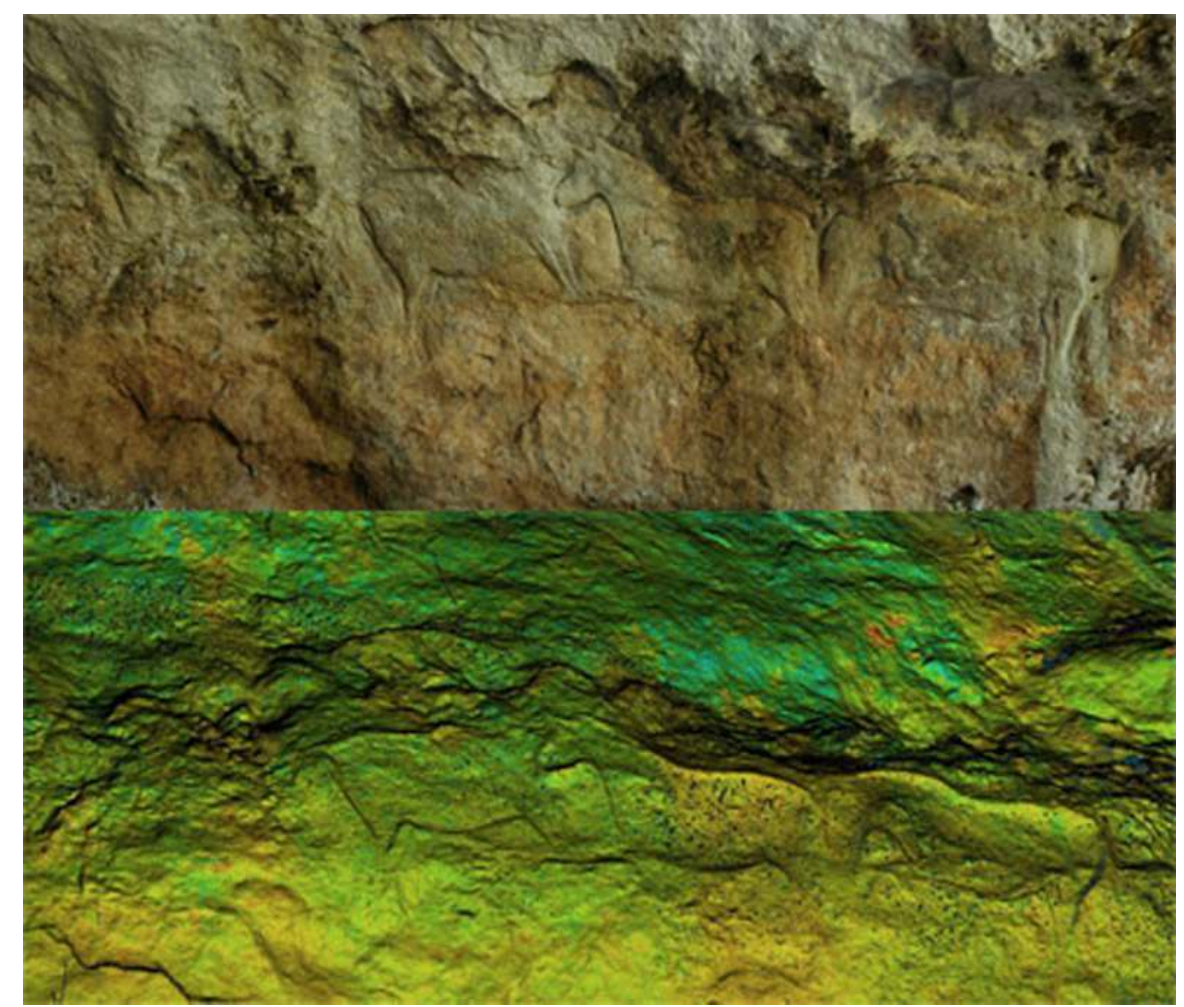

Frise sculptée de la Chaire-à-Calvin : cliché et modèle numérique 3D

(C) Geneviève Pinçon, Conseil général de la Charente, base de données 3D Arts graphiques et patrimoine. Phot. Alain Maulny

3 L'enregistrement laser 3D répond à un cahier des charges fixant les objectifs et les besoins (spécifiques à chaque site) et déterminant ainsi le degré de précision de la saisie ${ }^{3}$. La problématique d'étude du Roc-aux-Sorciers a dicté trois niveaux de précision: de 2 à 5 millimètres pour les zones naturelles, de 5 à 8 dixièmes de millimètres pour les zones anthropiques sculptées, de 1 à 2 dixièmes de millimètres pour les zones anthropiques gravées.

4 Pour un usage scientifique pertinent des modèles numériques, des clichés photographiques de qualité ont été réalisés en parallèle pour l'habillage des bases de données. Ils ont été numérisés et enregistrés selon les normes préconisées par le ministère de la Culture ${ }^{4}$.

5 Le laser 3D livre un enregistrement fidèle et précis des œuvres gravées et sculptées, dans leurs formes, leurs dimensions et leur position relative réelle. Des distorsions peuvent apparaître lors de l'habillage du modèle numérique (mapping) lorsque des images en deux dimensions sont plaquées sur une surface en trois dimensions. À la différence des 
dessins et peintures, cette question ne se pose pas directement pour les œuvres en volume (sculpture, gravure), contenues et accessibles dans le modèle numérique brut.

\section{Une approche affinée de la volumétrie : mesures, plans et coupes}

6 Plus encore que l'enregistrement, les applications offertes par les technologies tridimensionnelles constituent leur grande originalité.

Figure 3

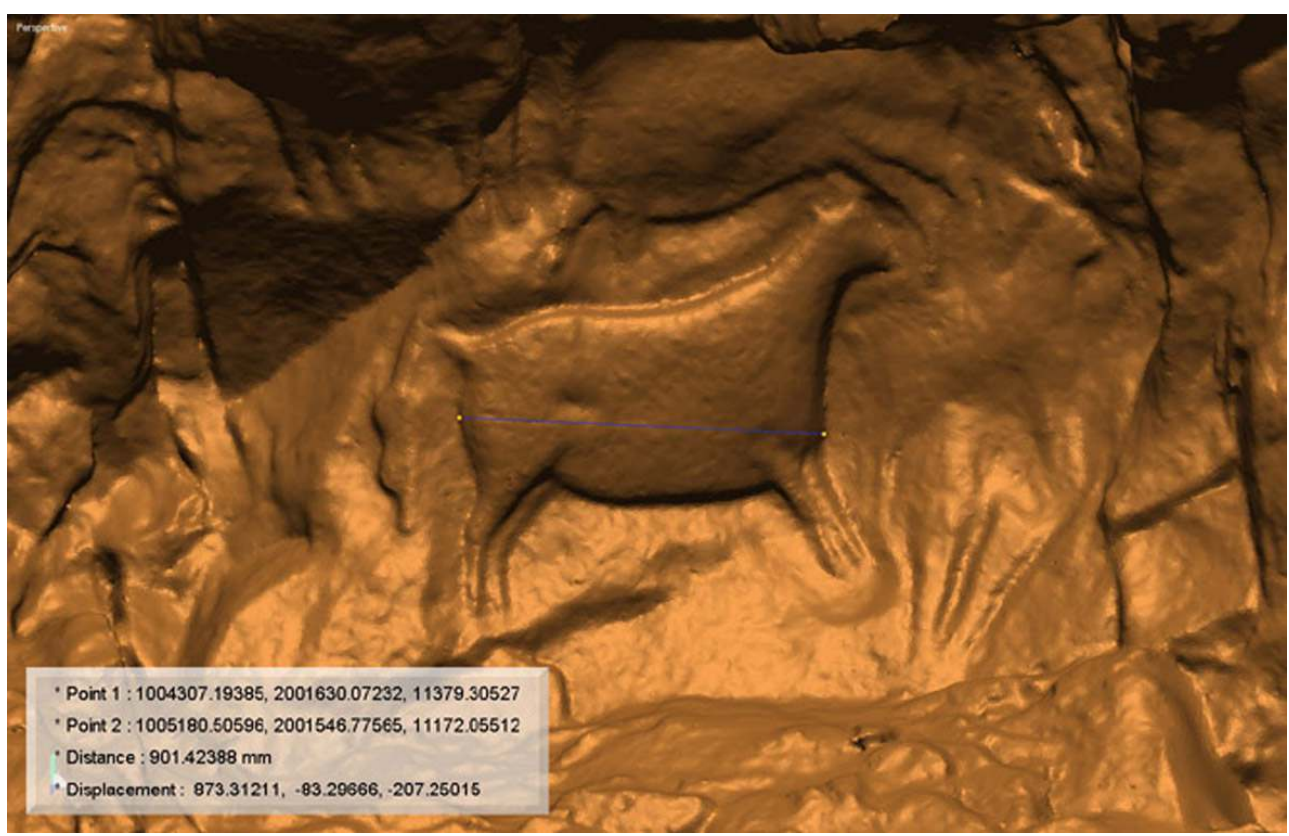

Prise de mesures sur l'objet 3D : longueur du corps du bouquetin femelle du Roc-aux-Sorciers (Abri Bourdois, panneau des bouquetins)

(C) Geneviève Pinçon. Ministère de la Culture et de la Communication, base de données 3D Arts graphiques et patrimoine

Des mesures, des coupes et des profils de l'objet peuvent être aisément effectués directement sur le modèle numérique brut (nuages de points). Les mesures sont fiables et précises puisque l'orthogonalité est parfaitement respectée (fig. $\mathbf{n}^{\circ} \mathbf{3}$ ). En outre, les coupes et profils - qui demandent un certain investissement logistique et temporel sur le terrain - sont ici dressés autant que de besoin, suivant un rythme déterminable (fig. $\mathbf{n}^{\circ}{ }^{4}$ ) 


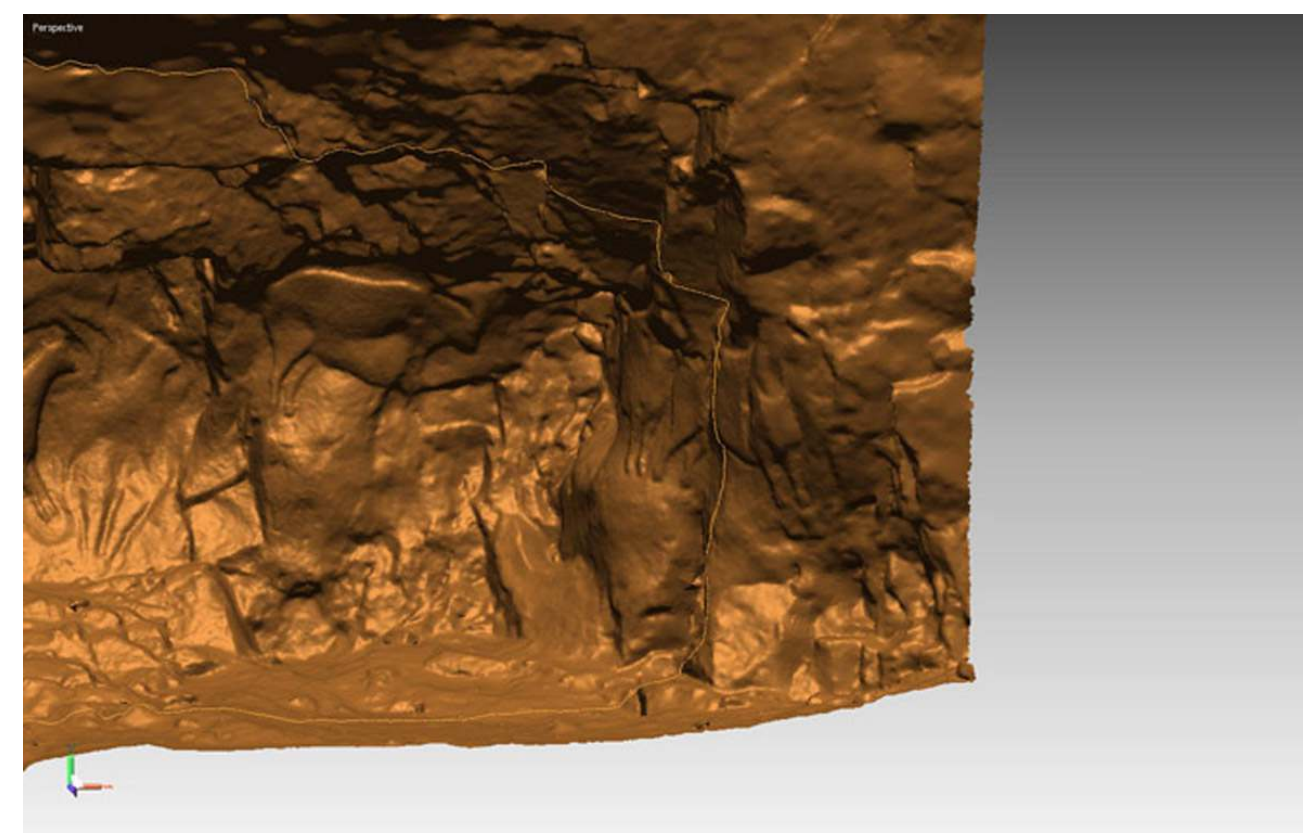

Tracé d'une coupe sur l'objet 3D : milieu du tronc du bouquetin n 8 du Roc-aux-Sorciers (Abri Bourdois, panneau des bouquetins)

Doc. Geneviève Pinçon. (c) Geneviève Pinçon. Ministère de la Culture et de la Communication, base de données 3D Arts graphiques et patrimoine

Ces outils 3D offrent une approche affinée de la volumétrie de la paroi et des œuvres. Ils permettent d'étudier la morphologie du support et d'apprécier son rôle dans la réalisation des figures (intégration d'éléments naturels à la représentation), ou encore les modifications anthropiques qu'il a pu subir (préparation de la paroi avant exécution des œuvres). Ils se révèlent également précieux dans l'analyse de la morphométrie des figurations, participant à la définition de modèles artistiques à travers l'affirmation de canons de représentation. Enfin, les coupes et profils des tracés contribuent à l'étude technique des œuvres, en aidant à la détermination de l'outil employé, ainsi qu'à l'établissement de leur chronologie relative, par l'observation des superpositions.

9 Les outils 3D permettent ainsi de travailler à différentes échelles sur le modèle numérique et d'aborder une multitude de problématiques.

\section{Tester et simuler des hypothèses en temps réel}

L'apport majeur des technologies 3D réside dans les multiples simulations qu'elles permettent de développer. 
Figure 5

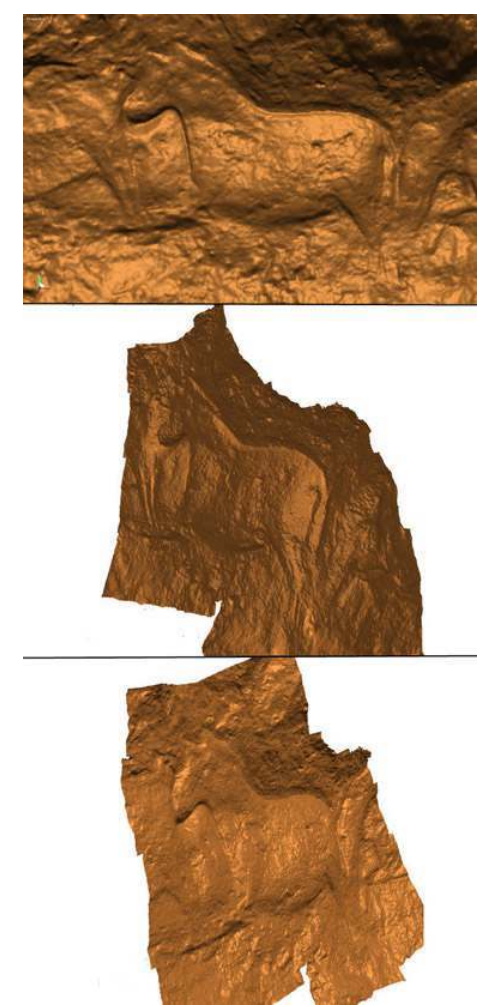

Simulation du changement de la position de l'observateur sur la sculpture centrale de la Chaire-àCalvin, vue frontale, vue de profil gauche, vue de profil droit

(C) Geneviève Pinçon. Ministère de la Culture et de la Communication, base de données 3D Arts graphiques et patrimoine

11 L'observateur peut se déplacer virtuellement autour de l'objet numérique, sous toutes ses faces, s'en approcher ou s'en détacher à volonté (fig. $\left.\mathbf{n}^{\circ} 5\right)$. Cette modification des angles d'observation est primordiale dans la lecture des œuvres pariétales, qu'elles soient en relief (sculptures) ou qu'elles utilisent le volume de la paroi, ce qui est très régulièrement le cas. Cet accès facilité et optimisé à l'œuvre vient fréquemment palier des difficultés d'observation dans les conditions réelles (figure plafonnante et distante dans ce cas). 
Figure 6

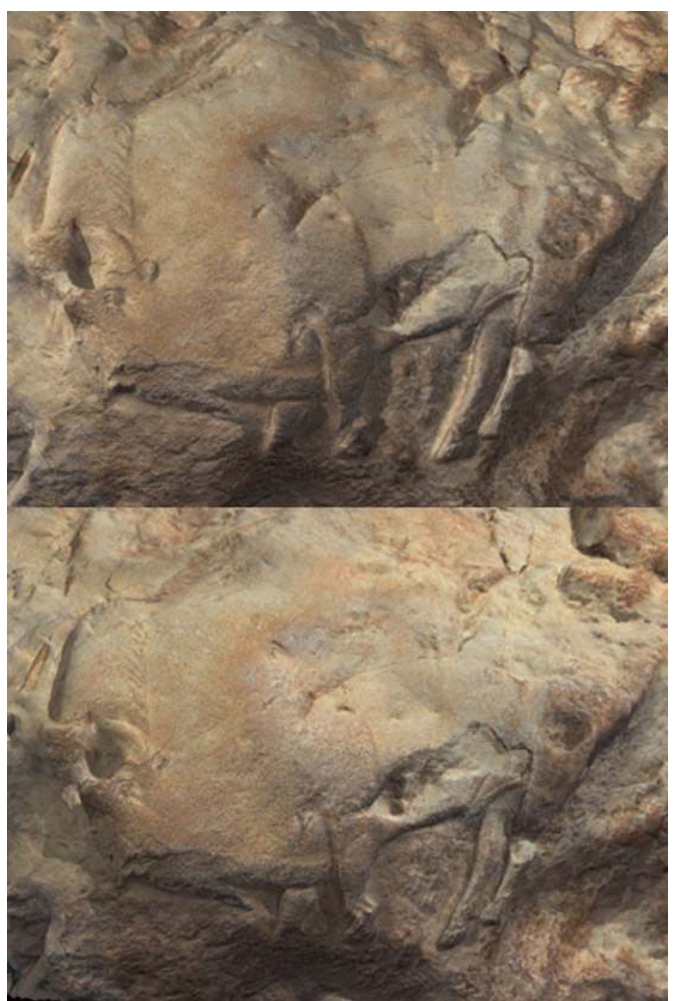

Simulation de la variation de la source lumineuse sur la sculpture de bison in situ de la Cave Taillebourg (Roc-aux-Sorciers), éclairage d'est, éclairage d'ouest

(C) Geneviève Pinçon. Ministère de la Culture et de la Communication, base de données 3D Arts graphiques et patrimoine

Le placement de la source lumineuse peut aussi être varié à l'infini. Comme la position de l'observateur, ce paramètre est essentiel dans la perception des œuvres, particulièrement des sculptures, qui jouent pleinement avec les effets d'ombre. Dans notre contexte artistique d'abris ornés, ce jeu de lumière a dû être prégnant dans la conception des figures, sur lesquelles l'évolution de l'éclairage solaire au cours de la journée et au cours de l'année (saisons) devait influer (fig. $\mathbf{n}^{\circ}$ 6). De même, les éclairages nocturnes avec la simulation des foyers retrouvés en fouilles complètent l'approche de ces œuvres dans leur contexte d'habitat ${ }^{5}$.

Dans certains cas, cette simulation comble à nouveau une lacune de terrain. Au Roc-auxSorciers, la frise a été mise au noir. Elle ne peut plus désormais être vue comme les hommes du Paléolithique l'ont perçue, imaginée et créée.

Des raccords virtuels de blocs ornés peuvent être entrepris (fig. $\mathbf{n}^{\circ} 7$ ). Ils sont automatiquement calculés et réalisés par l'ordinateur, à partir des points de calage sur les pans de fracture et vérifiés ensuite par le degré d'adhérence par rapport à un raccord idéal. 
Figure 7

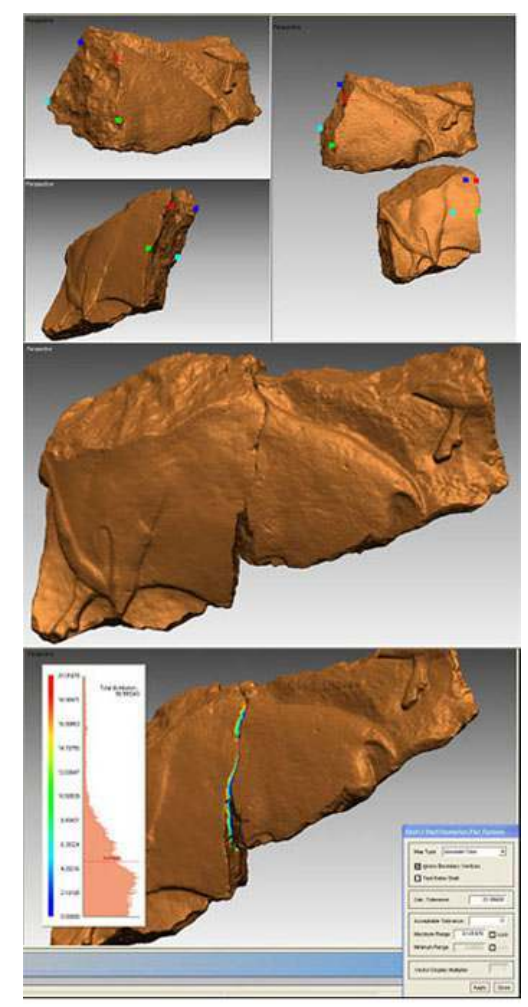

Raccord de deux fragments d'une sculpture de bison du Roc-aux-Sorciers (Cave Taillebourg), calcul et sélection des points de calage par l'ordinateur, raccord des modèles numériques, vérification des ajustements

(C) Geneviève Pinçon. Ministère de la Culture et de la Communication, base de données 3D Arts graphiques et patrimoine

Cette application s'avère particulièrement intéressante au Roc-aux-Sorciers dont plusieurs dizaines de mètres carrés de voûte ornée se sont effondrés et brisés en quelques centaines de blocs, pour la plupart difficilement manipulables en raison de leur poids ou de leur stockage dans des espaces confinés. 
Figure 8

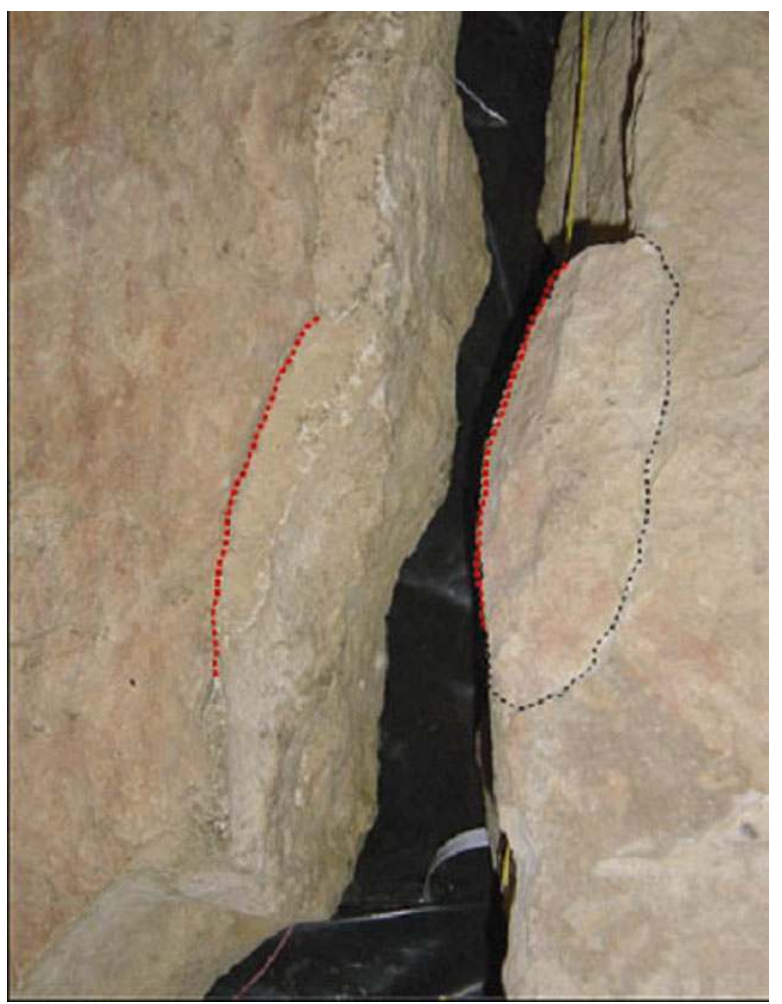

Raccord de deux blocs peints et gravés du Roc-aux-Sorciers, affectant une arête (Abri Bourdois)

(c) Geneviève Pinçon. Ministère de la Culture et de la Communication, phot. Geneviève Pinçon

Néanmoins, les raccords numériques connaissent quelques limites. L'opérateur doit en effet cadrer l'ordinateur dans ses tests, en le restreignant aux seules surfaces bordant la face décorée. Il aura autrement tendance à étudier toutes les possibilités, ce qui rallongerait considérablement le temps de résolution. En outre, l'ordinateur peine à calculer un raccord quand il n'affecte qu'une arête de fracture, et non un pan entier (fig. $\mathbf{n}^{\circ} \mathbf{8}$ ). Si le raccord numérique constitue un remarquable outil d'étude, l'expérience a montré l'intérêt d'employer des modèles réduits, tirés à partir du modèle numérique tridimensionnel, pour tenter des raccords physiques, à la manière d'autres types de mobilier archéologique (industrie lithique, céramique...).

Les comparaisons de formes (homothéties) sur les modèles tridimensionnels permettent d'appréhender avec beaucoup plus de rigueur et de justesse les marges de différence entre deux œuvres, en particulier entre sculptures ${ }^{6}$. 
Figure 9

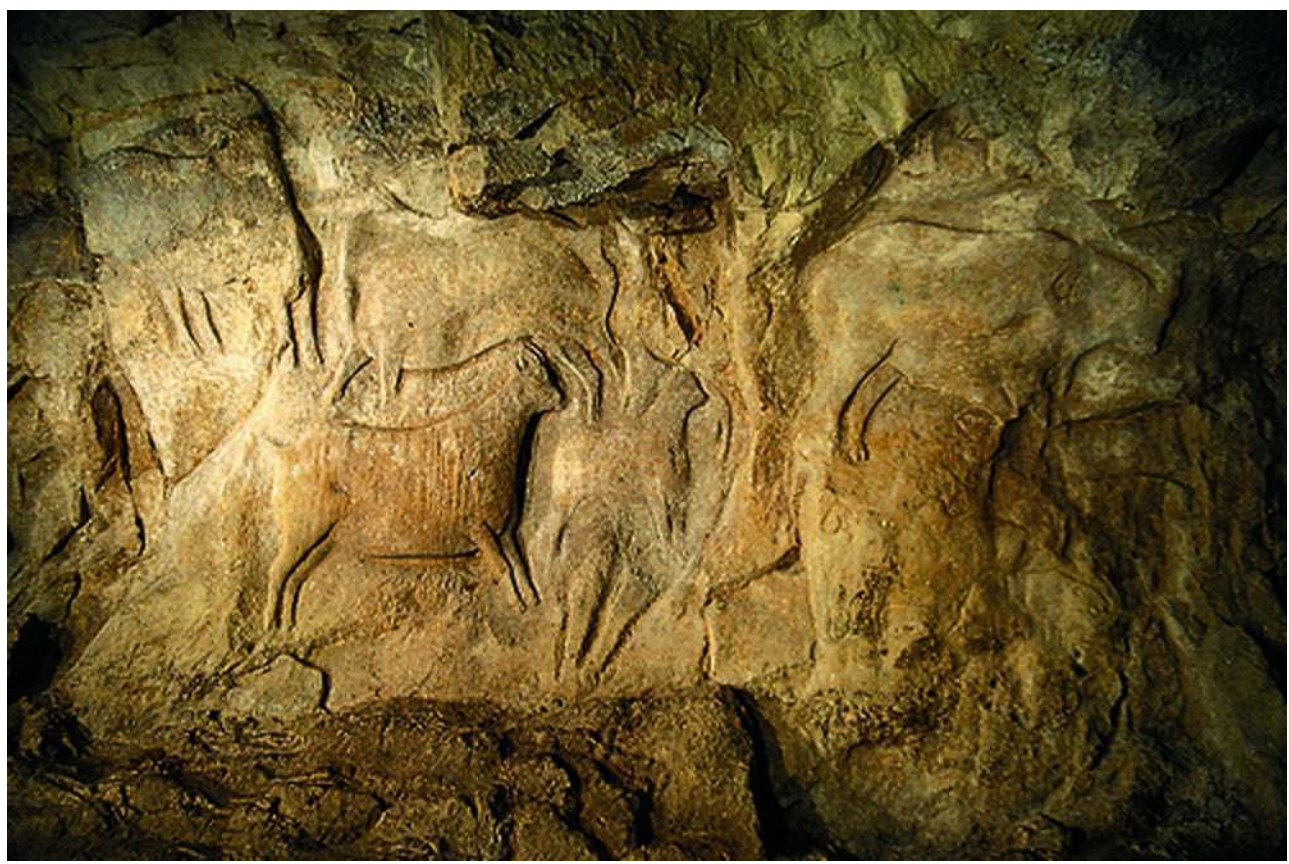

Vue frontale du panneau des bouquetins au Roc-aux-Sorciers (Abri Bourdois)

(C) Geneviève Pinçon. Ministère de la Culture et de la Communication. Phot. Geneviève Pinçon

La frise du Roc-aux-Sorciers comporte diverses sculptures partielles (réduites à quelques segments corporels), vestiges d'anciennes représentations en partie détruites au profit de nouvelles, repérés lors du travail de relevé analytique graphique en $2 \mathrm{D}^{7}{ }^{8}$. Dans l'Abri Bourdois, le panneau des bouquetins regroupe cinq figures de bouquetins, réparties en deux registres (fig. $\mathbf{n}^{\circ}$ 9): deux bouquetins mâles surplombent une tête de vieux bouquetin, une femelle et un chevreau, associés à la partie inférieure d'une représentation féminine (sexe et jambes) sous le chevreau et à un arrière-train d'animal (croupe, queue et fesse) devant le chevreau. Enfin, un sabot apparait dans le dessin du sexe de la femme, intégré dans la reproduction de la lèvre droite. 
Figure 10

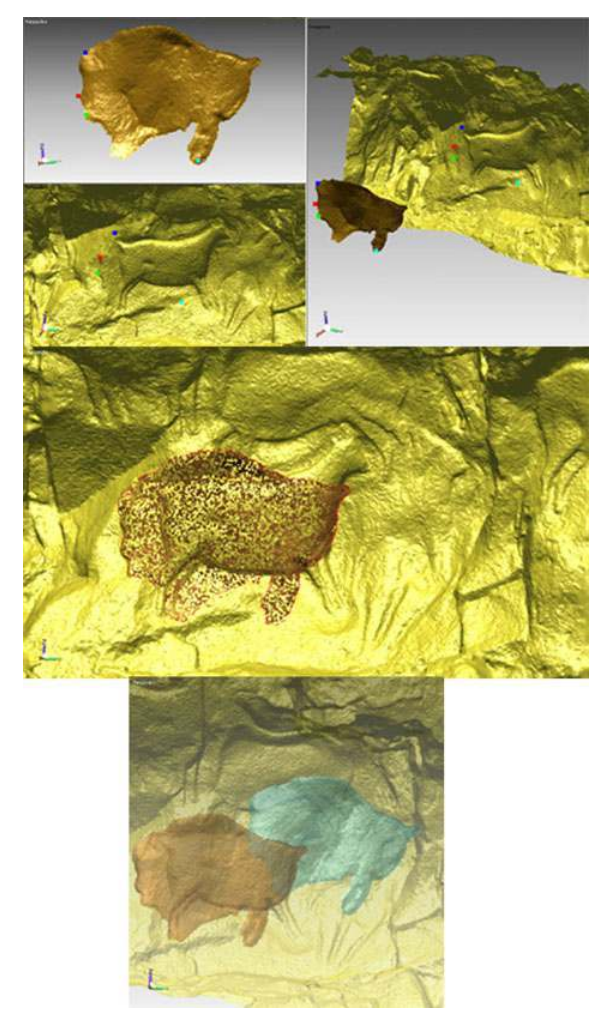

Interprétation de figures partielles du panneau des bouquetins (Roc-aux-Sorciers, Abri Bourdois) à l'aide de la bibliothèque de formes 3D, découpage de la figure et sélection des points de calage, superposition de la tête de vieux bouquetin et d'une sculpture de bison de la frise, superposition de l'arrière-train indéterminé et d'une sculpture de bison de la frise

Doc. Geneviève Pinçon. (c) Geneviève Pinçon, Ministère de la Culture et de la Communication, base de données 3D Arts graphiques et patrimoine

19 Au regard de la queue longue et fine, l'arrière-train pourrait être celui d'un bison ou d'un aurochs. L'aurochs est, dans l'état actuel des connaissances, absent de la frise. La projection d'une sculpture de bison mâle de la frise sur ce vestige, en adaptant l'orientation et les dimensions, est saisissante (fig. $\mathbf{n}^{\circ} \mathbf{1 0}$ ): les deux arrière-trains se confondent et le sabot postérieur droit se superpose parfaitement au sabot signalé dans le sexe de la femme. En outre, la ligne cervico-dorsale vient longer un relief sinueux situé à l'intérieur de l'un des deux bouquetins mâles du registre supérieur. Ce relief avait été considéré en premier lieu comme un volume interne propre à la représentation du bouquetin. Il s'agit en fait de la contre-empreinte du corps du bison effacé lors de la réalisation du bouquetin mâle, de la femelle et du chevreau.

La tête de vieux bouquetin paraissait assez ambiguë à S. de Saint-Mathurin qui y voyait également celle d'un bison'. En effet, les deux animaux possèdent des profils très proches : tous deux sont pourvus d'une barbiche, la morphologie des cornes du bouquetin peut être assimilée à la bosse dorsale du bison. La projection d'une sculpture de bison mâle de la frise sur cette œuvre, en adaptant l'orientation, est frappante (voir fig. $\mathbf{n}^{\circ} \mathbf{1 0}$ ) : les profils correspondent en tous points. En outre, les sabots des pattes postérieures viennent se loger dans un petit creusement piqueté interprété comme une ancienne attache d'extrémité de pattes, situé juste sous le ventre de la femelle et qui restait, jusqu'alors, inexpliqué. Un premier bison a donc été partiellement détruit pour la 
réalisation de l'étagne (bouquetin femelle), et probablement du second bouquetin mâle du registre supérieur.

Ces hypothèses avaient déjà été évoquées et testées avec les relevés 2D. Cependant, l'intérêt des outils 3D est d'appliquer des volumes venant asseoir notre démonstration. Ils aident à l'interprétation de figures partielles, et ainsi à l'établissement de la chronologie interne d'un décor pariétal en proposant une modélisation plausible des différentes étapes de la frise.

Ces comparaisons homothétiques peuvent concerner des œuvres provenant de sites différents, dans la problématique des relations inter-sites et plus généralement des territoires artistiques.

Des essais ont été menés entre diverses sculptures du Roc-aux-Sorciers et de la Chaire-àCalvin. Les deux ensembles pariétaux ont livré lors de leur relevé de nombreuses similitudes touchant à la technique utilisée, aux thèmes représentés, aux dimensions des figures, à leur traitement formel (morphologie générale, choix et rendu des détails anatomiques) et aux attitudes qui leur ont été données (tête baissée...).

Figure 11

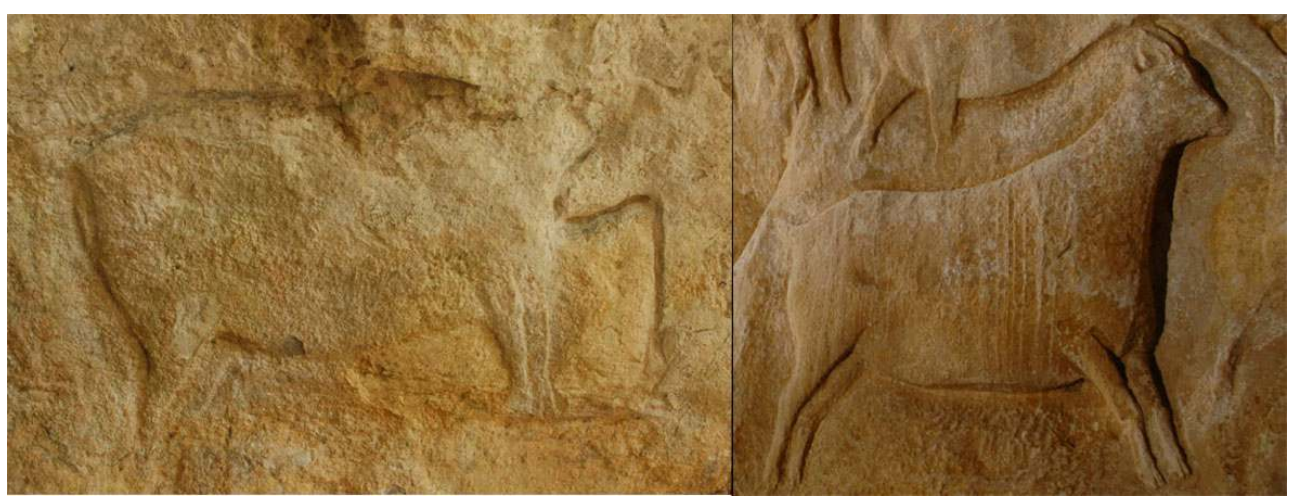

Mise en parallèle de l'animal acéphale de la Chaire-à-Calvin et du bouquetin femelle du Roc-auxSorciers (Abri Bourdois, panneau des bouquetins)

(c) Geneviève Pinçon, Conseil général de la Charente, phot. Alain Maulny. (c) Geneviève Pinçon. Ministère de la Culture et de la Communication, phot. Geneviève Pinçon

Nous avons notamment choisi de mettre en parallèle l'animal acéphale de la Chaire-àCalvin - décrit comme bison ${ }^{10}$, bovidé ${ }^{11}{ }^{12}$, aurochs ${ }^{13}$ et cheval ${ }^{14}$ et le bouquetin femelle du Roc-aux-Sorciers (fig. $\mathbf{n}^{\circ} \mathbf{1 1}$ ). Les deux animaux possèdent en effet des silhouettes semblables : tronc épais, pattes courtes et minces, représentées par paires, et surtout un même détail relativement peu figuré dans l'art pariétal paléolithique : la vulve. L'animal acéphale est apparemment dépourvu de queue, certainement détruite par l'importante érosion subie par la sculpture. Tous deux sont campés sur leurs membres, joints et tendus.

La figure du bouquetin femelle est découpée dans le modèle numérique du Roc-auxSorciers et amenée dans celui de la Chaire-à-Calvin, où elle est projetée sur l'animal acéphale, sans toucher aux dimensions mais en ajustant l'orientation (fig. $\mathbf{n}^{\circ} \mathbf{1 2}$ ). Le flanc, les pattes antérieures et le poitrail concordent. L'arrière-train du bouquetin femelle est, en revanche, plus court. 
Figure 12

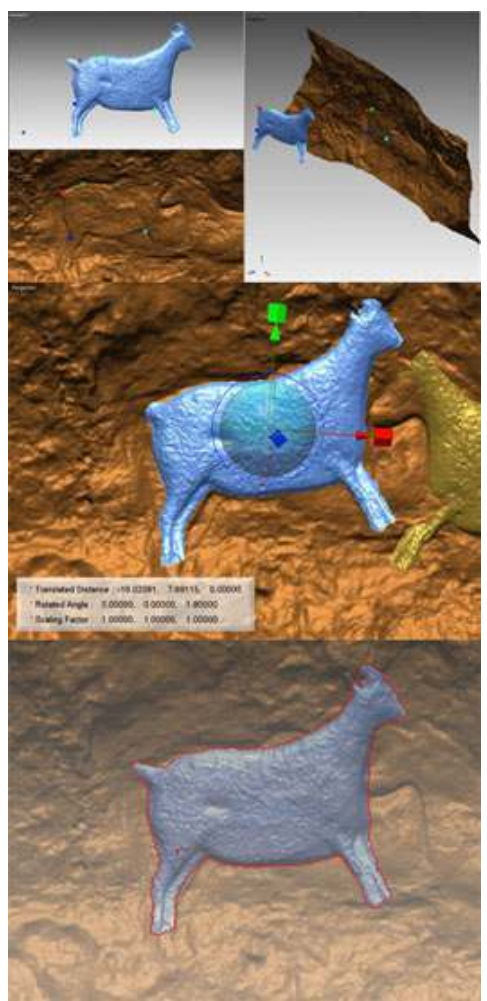

Comparaison homothétique entre l'animal acéphale de la Chaire-à-Calvin et le bouquetin femelle du Roc-aux-Sorciers (Abri Bourdois, panneau des bouquetins), découpage de la figure et sélection des points de calage, superposition avec ajustement de l'orientation pour une comparaison optimale

Doc. Geneviève Pinçon. (c) Geneviève Pinçon, Conseil général de la Charente, base de données 3D Arts graphiques et patrimoine

À nouveau, les outils 3D permettent d'appuyer nos hypothèses quant à la nature d'œuvres incomplètes, ici probablement un bouquetin, par la prise en compte de la volumétrie et non plus seulement des formes (dans le cas des relevés 2D). Ils offrent, de plus, la possibilité de préciser le degré de proximité entre deux représentations en quantifiant leurs marges de différence. Dans notre exemple, la question de l'auteur des deux sculptures et du groupe ayant fréquenté à un moment ces deux lieux distants de plus d'une centaine de kilomètres est posée.

\section{Des approches scientifiques approfondies}

Les technologies 3D enrichissent considérablement nos analyses. Leurs applications viennent approfondir notre démarche scientifique en art pariétal paléolithique sur des questions jusqu'à présent excessivement difficiles à traiter (raccords de blocs, liens entre sites pariétaux). Elles s'avèrent particulièrement appropriées aux études sur la sculpture.

(fig. $\left.n^{\circ} 13\right)$

Les outils tridimensionnels possèdent aussi quelques limites, notamment méthodologiques. En effet, dans le cadre des comparaisons homothétiques, la précision des résultats est fonction de la définition du modèle numérique manipulé. Le découpage des figures demeure en outre complètement subjectif, laissé à l'appréciation du 
chercheur et de sa lecture de l'œuvre. L'enregistrement 3D ne remplace d'ailleurs pas le relevé analytique, seul le travail d'analyse permettant de cerner l'œuvre en distinguant le naturel de l'anthropique.

Figure 13

\section{inp Institut national du patrimoine}

Communication présentée lors du séminaire ART RUPESTRE : LA 3D UN OUTIL DE MÉDIATION DU RÉEL INVISIBLE ? qui s'est tenu du 4 au 6 juin 2008 à Angles-sur-l'Anglin, coordonné par l'Institut national du patrimoine, avec la participation de la direction de l'architecture et du patrimoine

Enfin, pour être intéressant et efficient, le recours aux images 3D en art pariétal paléolithique doit intégrer des problématiques clairement définies.

\section{NOTES}

1. Pinçon, Geneviève. Méthodes de relevé appliquées à l'étude de l'art pariétal sculpté magdalénien du Roc-aux-Sorciers (Angles-sur-l'Anglin, France), Bulletin de l'Association des archéologues de Poitou-Charentes, 2004, n³3, 2004, p. 41-58.

2. Pinçon, Geneviève, Fuentes, Oscar, Bourdier, Camille, Hamon, Gaël. Technologies 3D appliquées à la sculpture pariétale magdalénienne, Images et relevés archéologiques, de la preuve à la démonstration, sous la direction d'Olivier Buchsenchutz, 132e congrès national des sociétés savantes historiques et scientifiques, Arles, 2007. Paris : Éditions du CTHS, 2007. CD Rom, 2008.

3. Voir le site : http://www.artgp.fr/realisations/angles/index.html.

4. Voir le site : http://www.culture.gouv.fr/culture/mrt/numerisation/fr/f_04.htm.

5. Voir le site : http://www.roc-aux-sorciers.com/bande_annonce.php.

6. Pinçon, Geneviève, Bourdier, Camille, Fuentes, Oscar. Les sculptures pariétales magdaléniennes du Roc-aux-Sorciers (Vienne) et de la Chaire-à-Calvin (Charente) : œuvres d'un groupe culturel ou d'un seul et même artiste? Virtual Restrospect 2007, Actes du colloque Pessac (Gironde), 14-16 novembre 2007, collection Archéovision, 2008, volume 3. Bordeaux: Ausonius édition, 2008, p. 13-20.

7. Pinçon, Geneviève, Iakovleva, Ludmila. La frise sculptée du Roc-aux-Sorciers, Angles-surl'Anglin. Paris : Éditions du CTHS-RMN, 1997.

8. Pinçon, Geneviève. Chronologie pariétale des œuvres magdaléniennes du Roc-aux-Sorciers (Angles-sur-l'Anglin, Vienne) : entre tradition et innovation. In Situ, revue des patrimoines [document électronique], 2008, n 9. Paris : Ministère de la Culture et de la Communication.

9. Saint-Mathurin, Suzanne de. L'abri du Roc-aux-Sorciers. In L'art des Cavernes. Atlas des grottes ornées paléolithiques françaises, ministère de la Culture. Paris : Imprimerie Nationale, 1984, p. 583-587.

10. David, Pierre. Frise de l'abri sous roche dit " de la Chaire à Calvin » ou de la "Papeterie ", commune de Mouthiers (Charente). In Congrès de l'Association Française pour l'Avancement des Sciences, 53e session, Le Havre, 1929, p. 1-4. 
11. David, Pierre. Station solutréenne de Mouthiers (Charente). Société Linnéenne de Bordeaux , 1929.

12. Bouvier, Jean-Marc. La Chaire-à-Calvin (Mouthiers, Charente). Données et problèmes. In IX congrès de l'UISPP, Nice, 1976, p. 133-136.

13. Leroi-Gourhan, André. Préhistoire de l'art occidental. Paris : Mazenod, 1965, p. 491-493.

14. Sonneville-Bordes, Denise de. Étude de la frise sculptée de la Chaire-à-Calvin. Commune de Mouthiers (Charente), Annales de Paléontologie, 1963, tome XLIX, p. 81-193.

\section{RÉSUMÉS}

Si les technologies 3D livrent un enregistrement précis et pertinent des graphismes pariétaux, elles offrent également des applications particulièrement intéressantes pour leur analyse. À travers des traitements sur nuage de points et des simulations, elles autorisent un large éventail de manipulations touchant autant à l'observation qu'à l'étude des œuvres pariétales. Elles permettent notamment une perception affinée de leur volumétrie, et deviennent des outils de comparaison de formes très utiles dans la reconstruction des chronologies pariétales et dans l'appréhension des analogies entre différents sites. Ces outils analytiques sont ici illustrés par les travaux originaux menés sur les sculptures pariétales des abris du Roc-aux-Sorciers (Angles-surl'Anglin, Vienne) et de la Chaire-à-Calvin (Mouthiers-sur-Boëme, Charente).

If 3D technologies allow an accurate and relevant recording of rock art, they also offer several interesting applications for its analysis. Through spots clouds treatments and simulations, they permit a wide range of manipulations concerning figurations observation and study. Especially, they allow a fine perception of their volumetry. They become efficient tools for forms comparisons, very useful in the reconstruction of graphic ensemble chronologies and for intersites analogies. These analytical tools are illustrated by the original works done on the sculptures of Roc-aux-Sorciers (Angles-sur-l'Anglin, Vienne) and Chaire-à-Calvin (Mouthiers-sur-Boëme, Charente) rock-shelters.

\section{INDEX}

Mots-clés : art pariétal, analyse, simulation, sculpture, chronologie pariétale, homothétie, volumétrie, comparaisons inter-sites, Roc-aux-Sorciers, Chaire-à-Calvin

Keywords : rock art, analysis, graphic ensemble chronology, homothety, volumetry, inter-sites comparisons

\section{AUTEURS}

\section{GENEVIÈVE PINÇON}

Ministère de la Culture et de la Communication, Direction de l'architecture et du patrimoine, chercheur UMR ArScan 7041 ; Musée d'archéologie nationale. genevieve.pincon@culture.gouv.fr 


\section{CAMILLE BOURDIER}

Doctorante, PACEA UMR 5199, Université Bordeaux 1, Institut de Paléontologie et de Géologie du Quaternaire.ca.bourdier@laposte.net

\section{OSCAR FUENTES}

Doctorant, UMR ArScan 7041, Université Paris 1, Maison de l'Archéologie et de l'Ethnologie.

fuentes.osc@gmail.com

\section{AURÉLIE ABGRALL}

Doctorante, UMR 7055 Préhistoire et Technologie, Université Paris 10-Paris-Nanterre.

aurelie.abgrall@club-internet.fr 